\title{
Produção de biodiesel de terceira geração a partir de microalgas
}

\author{
Third generation biodiesel production from microalgae
}

\author{
Érika Cristina Francisco ${ }^{\mathrm{I}, \text { II }}$ Telma Teixeira Franco ${ }^{\mathrm{II}}$ Mariana Manzoni Maroneze $^{\mathrm{III}}$ \\ Leila Queiroz Zepka ${ }^{\text {III }}$ Eduardo Jacob-Lopes ${ }^{\text {II* }}$
}

\section{RESUMO}

$O$ objetivo do estudo foi avaliar o biodiesel de $3 \stackrel{a}{a}$ geração produzido a partir do cultivo heterotrófico da microalga Phormidium sp., empregando amido de mandioca como fonte de carbono orgânico. Um planejamento experimental foi realizado para determinar as condições ótimas de temperatura e razão C/N. A partir da obtenção das melhores condições de cultivo desenvolveram-se cultivos em batelada e batelada alimentada em biorreator e avaliaram-se as características do biodiesel produzido. Os resultados indicaram que a temperatura de $30^{\circ} \mathrm{C}$ e a razão $C / N$ de 68 são as condições ideais do processo. As maiores produtividades em biomassa $\left(50,41 \mathrm{mgL}^{-1} \mathrm{~h}^{-1}\right)$ e lipídica $\left(7,49 \mathrm{mgL}^{-1} h^{-1}\right)$ foram obtidas no cultivo em batelada. Os ácidos graxos com maior representatividade foram os ácidos caproico (65,29\%) e oleico $(23,88 \%)$. As propriedades de combustão do biodiesel: conteúdo de ésteres $(99,9 \%)$, número de cetano $(54,88)$, índice de iodo $\left(21,47 \mathrm{gI}_{2} 100 \mathrm{~g}^{-1}\right)$, grau de instauração $(23,88 \%)$ e ponto de entupimento de filtro a frio $\left(39,21^{\circ} \mathrm{C}\right)$ se mostraram adequadas às principais normativas nacionais e internacionais.

Palavras-chave: biodiesel, microalgas, cultivo heterotrófico, amido de mandioca.

\section{ABSTRACT}

The aim of the study was to evaluate the third generation biodiesel produced from heterotrophic cultivation of the microalgae Phormidium sp. employing cassava starch as source of organic carbon. An experimental design was performed to determine the optimal conditions of temperature and $C / N$ ratio. From the best growing conditions, was developed cultivations in batch and fedbatch in a bioreactor and evaluated the biodiesel quality. The results indicate that the temperature of $30^{\circ} \mathrm{C}$ and the $\mathrm{C} / \mathrm{N}$ ratio of 68 are the ideal conditions of the process. The highest biomass productivity $\left(50.41 \mathrm{mg}^{-1} h^{-1}\right)$ and lipid productivity $\left(7.49 \mathrm{mgL}^{-1} \mathrm{~h}^{-1}\right)$ were obtained in batch cultivations. The fatty acids most representative were caproic acid (65.29\%) and oleic acid (23.88\%). The fuel properties of biodiesel: ester content of $99.9 \%$, cetane number of 54.88 , iodine

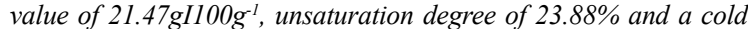
filter plugging point of $39.21^{\circ} \mathrm{C}$, comply with the main international and national standards.

Key words: biodiesel, microalgae, heterotrophic cultivation, cassava starch.

\section{INTRODUÇÃO}

A grande dependência pelos combustíveis fósseis levou ao surgimento de discussões sobre questões como a poluição atmosférica e a escassez dos recursos energéticos, direcionando ao desenvolvimento de tecnologias para a obtenção de fontes de energias alternativas e renováveis, como os biocombustíveis de 1a a 4a geração (MARTÍN \& GROSSMANN, 2012). Os biocombustíveis de $3^{\mathrm{a}}$ geração são obtidos a partir do cultivo de microalgas, que possuem a capacidade de acumular lipídeos nas células, os quais, após extração, são transesterificados para a obtenção do biodiesel (MATA et al., 2010; HADDAD \& FAWAZ, 2013).

As microalgas estão classificadas em um grande grupo de algas microscópicas fotossintetizantes. Algumas espécies apresentam obrigatoriamente o metabolismo fotossintético, embora outras sejam capazes de crescer sob condições

ICurso de Engenharia Ambiental, Faculdade de Engenharia e Arquitetura, Universidade de Passo Fundo (UPF), Passo Fundo, RS, Brasil. "Faculdade de Engenharia Química, Universidade Estadual de Campinas (UNICAMP), Campinas, SP, Brasil.

IIIDepartamento de Tecnologia e Ciência dos Alimentos, Universidade Federal de Santa Maria (UFSM), 97105-900, Santa Maria, RS, Brasil. E-mail: jacoblopes@pq.cnpq.br. *Autor para correspondência. 
heterotróficas na total ausência de luminosidade e presença de uma fonte de carbono orgânico. Os sistemas heterotróficos superam algumas limitações dos fotossintéticos, como a dependência de luz, podendo viabilizar a produção de bioprodutos como lipídeos, proteínas, carboidratos e pigmentos (SUALI \& SARBATLY, 2012), além de serem conhecidos por resultarem em elevadas produtividades de bioprodutos (MOHAMED et al., 2011). Um fator determinante nos cultivos heterotróficos é a escolha da fonte de carbono orgânico utilizado, que representa um dos principais obstáculos para a aplicação industrial. $\mathrm{O}$ substrato orgânico é estimado em cerca de $80 \%$ do custo total do processo, podendo inviabilizar economicamente o processo. Uma alternativa aos elevados custos é a substituição de determinadas fontes de carbono orgânico por substratos de baixo custo, como amidos e soluções de celulose hidrolisada, podendo reduzir os custos em até 40\% (XU et al., 2006; LI et al., 2007). Além dos substratos orgânicos sintéticos, resíduos industriais têm sido considerados alternativas promissoras para a minimização do custo dos meios de cultura para o cultivo de microalgas (PEREZ-GARCIA et al., 2011; QUEIROZ et al., 2013).

Em face disso, o objetivo do estudo foi avaliar o biodiesel de 3 a geração produzido a partir do cultivo heterotrófico da microalga Phormidium sp., empregando amido de mandioca como fonte de carbono orgânico.

\section{MATERIAL E MÉTODOS}

Microrganismo e meio de cultura

A microalga utilizada foi a Phormidium sp., isolada do Deserto Cuatro Cienegas no México $\left(26^{\circ} 59^{\prime} \mathrm{N} 102^{\circ} 03 \mathrm{~W}\right)$. Após purificação, as culturas estoque foram mantidas em tubos de ensaio contendo meio sintético BG11 (RIPPKA et al., 1979) solidificado com agar-agar $\left(20 \mathrm{gL}^{-1}\right)$. As condições de manutenção foram $25^{\circ} \mathrm{C}$ e intensidade luminosa constante de $15 \mu \mathrm{molm}^{-2} \mathrm{~s}^{-1}$.

O meio sintético BG11 suplementado com amido de mandioca, como fonte de carbono orgânico exógeno foi empregado como meio de cultura. As diferentes razões carbono/nitrogênio $(\mathrm{C} / \mathrm{N})$ foram obtidas a partir da estequiometria do meio e de uma curva padrão construída com diferentes concentrações do amido de mandioca, expressa em termos de demanda química de oxigênio (DQO) (APHA, 2005).

\section{Planejamento experimental}

O planejamento experimental e as análises estatísticas foram realizados utilizando o software
Statistica 7.0 (STATSOFT, Tulsa, OK, USA). A partir de um delineamento composto central com cinco níveis e três repetições no ponto central, foi avaliada a relação entre as condições de cultivo (variáveis independentes) e os parâmetros cinéticos do processo (variáveis dependentes). As condições de cultivo estudadas foram temperatura $\left(23,25,30,35\right.$ e $\left.37^{\circ} \mathrm{C}\right)$ e razão $\mathrm{C} / \mathrm{N}(12,20,40,60$ e 68$)$ e como respostas avaliaram-se a produtividade de biomassa, a taxa de consumo do substrato, o coeficiente de conversão do substrato em células e a eficiência na conversão do carbono orgânico.

\section{Cultivo em Erlenmeyers}

A otimização do processo, mediante o planejamento experimental, foi realizada a partir do cultivo em frascos Erlenmeyers de $250 \mathrm{~mL}$, sendo o volume nominal de trabalho de $150 \mathrm{~mL}$, utilizando agitador orbital. Os frascos utilizados foram esterilizados em autoclave a $121^{\circ} \mathrm{C}$ por 20 minutos e a inoculação foi realizada assepticamente. As condições experimentais foram: meio sintético BG11 nas razões $\mathrm{C} / \mathrm{N}$ e temperatura, descritas pelo planejamento experimental, concentração inicial do inóculo de $100 \mathrm{mgL}^{-1}, \mathrm{pH}$ ajustado a 7,8 e agitação constante de $150 \mathrm{rpm}$.

\section{Cultivos em biorreatores}

Os cultivos em biorreator foram realizados em reator de coluna de bolhas construído em vidro borossilicato com um diâmetro externo de $12,5 \mathrm{~cm}$ e uma altura de $16 \mathrm{~cm}$, resultando numa relação altura/diâmetro (L/D) igual a 1,28. O volume total do frasco, bem como o volume nominal de trabalho, foi de 2,0L. O sistema de dispersão de ar consistiu em um difusor de $2,5 \mathrm{~cm}$ de diâmetro, localizado no interior do reator. A vazão de ar foi controlada por rotâmetros (precisão $\pm 5 \%$ ), a entrada de oxigênio e a saída dos gases foram filtradas através de unidades filtrantes Millex-FG ${ }^{\circledR}$ de $0,22 \mu \mathrm{m}$ de diâmetro.

Operacionalmente, dois modos de operação foram avaliados: modo descontínuo e descontínuo alimentado. As condições de cultivos foram: concentração celular inicial de $100 \mathrm{mgL}^{-1}$, aeração constante de 1,0VVM (volume de ar por volume de meio por minuto), $\mathrm{pH}$ ajustado a 7,8 , temperatura de $30^{\circ} \mathrm{C}$ e ausência de luminosidade. Adotou-se uma razão $\mathrm{C} / \mathrm{N}$ de 68 pela adição de $14,0 \mathrm{gL}^{-1}$ do amido de mandioca ao meio BG11. As alimentações nos cultivos descontínuos alimentados foram realizadas assim que a concentração de carbono orgânico atingisse $6,0 \mathrm{gL}^{-1}$. A alimentação foi realizada por meio de uma solução concentrada do amido $\left(7,0 \mathrm{gL}^{-1}\right)$, 
a fim de ajustar a concentração de carbono orgânico do meio à concentração de $14,0 \mathrm{gL}^{-1}$.

Amostragem e métodos analíticos

As amostragens foram realizadas de forma asséptica a cada 24 horas durante a fase de crescimento do microrganismo. A dinâmica do $\mathrm{pH}$ para os cultivos em biorreator foi determinada por potenciômetro e a concentração celular através de gravimetria por meio da filtração de um volume conhecido em filtro $0,45 \mu \mathrm{m}$ de diâmetro. A concentração de carbono orgânico, expressa em termos de demanda química de oxigênio (DQO) foi determinada por método colorimétrico do refluxo fechado segundo metodologia proposta por APHA (2005).

Ao término do processo, a biomassa foi separada do meio de cultivo por decantação, seguido de centrifugação, secagem e trituração.

Para a extração de lipídeos totais da biomassa e produção do biodiesel, utilizou-se o método de BLIGH \& DYER (1959) modificado, levando-se em conta as proporções entre os solventes metanol, clorofórmio e água destilada (2:1:0.8). A saponificação foi realizada através de proporções entre metanol, ácido clorídrico e clorofórmio (10:1:1), seguida da esterificação do extrato lipídico, a partir da reação entre o hexano e o clorofórmio (4:1), através do método de HARTMAN \& LAGO (1976) modificado. A análise qualitativa e quantitativa do biodiesel foi realizada por cromatografia gasosa (CG) utilizandose o cromatógrafo Varian 3400CX (Varian, Palo Alto, CA, EUA). Os ácidos graxos foram identificados por comparação dos tempos de retenção a partir de um padrão (Supelco, Louis, MO, EUA) e quantificados por normalização de área.

As propriedades de combustão do biodiesel foram determinadas de acordo com metodologia proposta por KRISNANGKURA (1986), RAMOS et al. (2008) e FRANCISCO et al. (2010). Avaliou-se o conteúdo de ésteres (EC), número de cetano (NC), índice de saponificação (IS), índice de iodo (II), o grau de instauração (GI), fator de comprimento da cadeia (FCC) e o ponto de entupimento de filtro a frio (PEFF), a partir de modelos empíricos que levam em consideração o perfil dos ácidos graxos presentes na matriz oleaginosa.

Análise dos dados cinéticos

Os dados de concentração de biomassa foram utilizados na obtenção da velocidade máxima específica de crescimento $\left(\ln \left(\mathrm{X} / \mathrm{X}_{0}\right)=\mu_{\max } \cdot \mathrm{t}\right)$, em que $\mathrm{X}$ é a concentração celular final $\left(\mathrm{mgL}^{-1}\right), \mathrm{X}_{0}$ é a concentração celular inicial $\left(\mathrm{mgL}^{-1}\right), \mu_{\max }$ é a velocidade máxima específica de crescimento $\left(\mathrm{h}^{-1}\right)$ e t é o tempo de residência $(\mathrm{h})$; no cálculo da produtividade de biomassa $\left(P_{X}=\mu . X\right)$, em que $\mu$ r a velocidade instantânea de crescimento $\left(\mathrm{h}^{-1}\right)$ e $\mathrm{X}$ a concentração celular $\left(\mathrm{mgL}^{-1}\right)$. A concentração de carbono orgânico foi utilizada para calcular a taxa de consumo do substrato $\left(r_{\mathrm{s}}=\mathrm{dS} / \mathrm{dt}\right)$, em que $\mathrm{S}$ é a concentração de carbono orgânico $\left(\mathrm{mgL}^{-1}\right)$ e t é o tempo de residência $(\mathrm{h})$; a eficiência na conversão do carbono orgânico $\left(E C=\mathrm{S}_{0}-\mathrm{S} / \mathrm{S}_{0}\right)$, em que $\mathrm{S}_{0}$ é a concentração inicial de carbono orgânico $\left(\mathrm{mgL}^{-1}\right)$ e S é a concentração final de carbono orgânico $\left(\mathrm{mgL}^{-1}\right)$ e o coeficiente de conversão do substrato em células $\left(\mathrm{Y}_{\mathrm{X} / \mathrm{S}}=\mathrm{dX} / \mathrm{dS}\right)$. Os experimentos foram realizados em duplicata e os dados cinéticos referem-se à média de quatro repetições.

\section{RESULTADOS E DISCUSSÃO}

Otimização do processo em frascos Erlenmeyer

A tabela 1 apresenta os parâmetros cinéticos de produção de biomassa e consumo de substrato nas diferentes condições de razão $\mathrm{C} / \mathrm{N}$ e temperatura, de acordo com o planejamento experimental. Observa-se, a partir da análise dos dados, uma variabilidade pronunciada no desempenho do processo em função dos fatores avaliados, obtendo-se produtividades em biomassa entre 6,33 a $50,72 \mathrm{mgL}^{-1} \mathrm{~h}^{-1}$, taxas de consumo de substrato entre 6,46 a $65,82 \mathrm{mgL}^{-1} \mathrm{~h}^{-1}$, coeficientes de conversão do substrato em células entre 0,17 a $0,81 \mathrm{mg}_{\text {célula }} \mathrm{mg}_{\text {amido }}{ }^{-1} \mathrm{e}$ conversões de carbono orgânico entre 10,08 a 99,61\%. Estes resultados, quando analisados a partir da figura 1, indicam que razões $\mathrm{C} / \mathrm{N}$ de 68 e temperaturas de $30^{\circ} \mathrm{C}$ favorecem a produção de biomassa microalgal. Comparativamente, a máxima produtividade em biomassa evidenciada neste estudo $\left(50,72 \mathrm{mgL}^{-}\right.$ ${ }^{1} \mathrm{~h}^{-1}$ ) é superior aos processos similares descritos na literatura, nos quais produtividades em biomassa na ordem de $36,0 \mathrm{mgL}^{-1} \mathrm{~h}^{-1}$ foram obtidas por $\mathrm{LU}$ et al. (2011), a partir do cultivo de Chlorella protothecoides em amido de mandioca hidrolisado.

Os coeficientes dos modelos, bem como o ajuste dos modelos de predição, validados a partir da distribuição F sugerem a existência de uma relação quadrática entre as variáveis, indicando a aderência aos dados experimentais.

\section{Cultivos em biorreator}

Com base nas condições definidas na etapa anterior, os cultivos em biorreator foram conduzidos mediante dois modos de operação distintos (batelada 
Tabela 1 -Matriz codificada do efeito da temperatura e razão $\mathrm{C} / \mathrm{N}$ para a produtividade de biomassa ( $\left.\mathrm{P}_{\mathrm{X}}\right)$, taxa de consumo do substrato ( $\mathrm{r}_{\mathrm{S}}$ ), coeficiente de conversão do substrato em células $\left(\mathrm{Y}_{\mathrm{X} / \mathrm{S}}\right)$ e eficiência na conversão do carbono orgânico (EC).

\begin{tabular}{|c|c|c|c|c|c|c|}
\hline Experimento & Temperatura & $\mathrm{C} / \mathrm{N}$ & $\mathrm{P}_{\mathrm{X}}\left(\mathrm{mgL}^{-1} \mathrm{~h}^{-1}\right)$ & $\mathrm{r}_{\mathrm{S}}\left(\mathrm{mgL}^{-1} \mathrm{~h}^{-1}\right)$ & $\mathrm{Y}_{\mathrm{X} / \mathrm{S}}\left(\mathrm{mg}_{\text {célula }} \mathrm{mg}_{\text {amido }}{ }^{-1}\right)$ & $\mathrm{EC}(\%)$ \\
\hline 1 & -1 & -1 & 14,5 & 25,24 & 0,96 & 38,52 \\
\hline 2 & +1 & -1 & 6,33 & 6,46 & 0,81 & 21,87 \\
\hline 3 & -1 & +1 & 31,46 & 50,47 & 0,86 & 30,97 \\
\hline 4 & +1 & +1 & 11,25 & 65,82 & 0,17 & 62,96 \\
\hline 5 & 0 & 0 & 15,59 & 17,32 & 0,70 & 10,51 \\
\hline 6 & 0 & 0 & 15,85 & 17,70 & 0,81 & 10,29 \\
\hline 7 & 0 & 0 & 15,72 & 17,51 & 0,75 & 10,08 \\
\hline 8 & $-1,41$ & 0 & 12,70 & 21,87 & 0,58 & 12,08 \\
\hline 9 & $+1,41$ & 0 & 24,66 & 53,41 & 0,46 & 72,63 \\
\hline 10 & 0 & $-1,41$ & 7,85 & 16,45 & 0,41 & 99,61 \\
\hline 11 & 0 & $+1,41$ & 50,72 & 55,47 & 0,67 & 49,43 \\
\hline
\end{tabular}

e batelada alimentada). Nesse sentido, os parâmetros cinéticos resultantes destes cultivos são apresentados na tabela 2 e figura 2. A análise dos dados indica que o cultivo em batelada apresentou taxas de consumo do substrato de $91,43 \mathrm{mgL}^{-1} \mathrm{~h}^{-1}$, eficiência de conversão do carbono orgânico de 75,19\%, concentração celular máxima de $4960 \mathrm{mgL}^{-1}$, eficiência de conversão de carbono orgânico de $75,19 \%$, produtividade de biomassa de $50,41 \mathrm{mgL}^{-1} \mathrm{~h}^{-1}$, teor de lipídeos de $14,86 \%$ e produtividade lipídica de $7,49 \mathrm{mgL}^{-1} \mathrm{~h}^{-1}$. Os cultivos

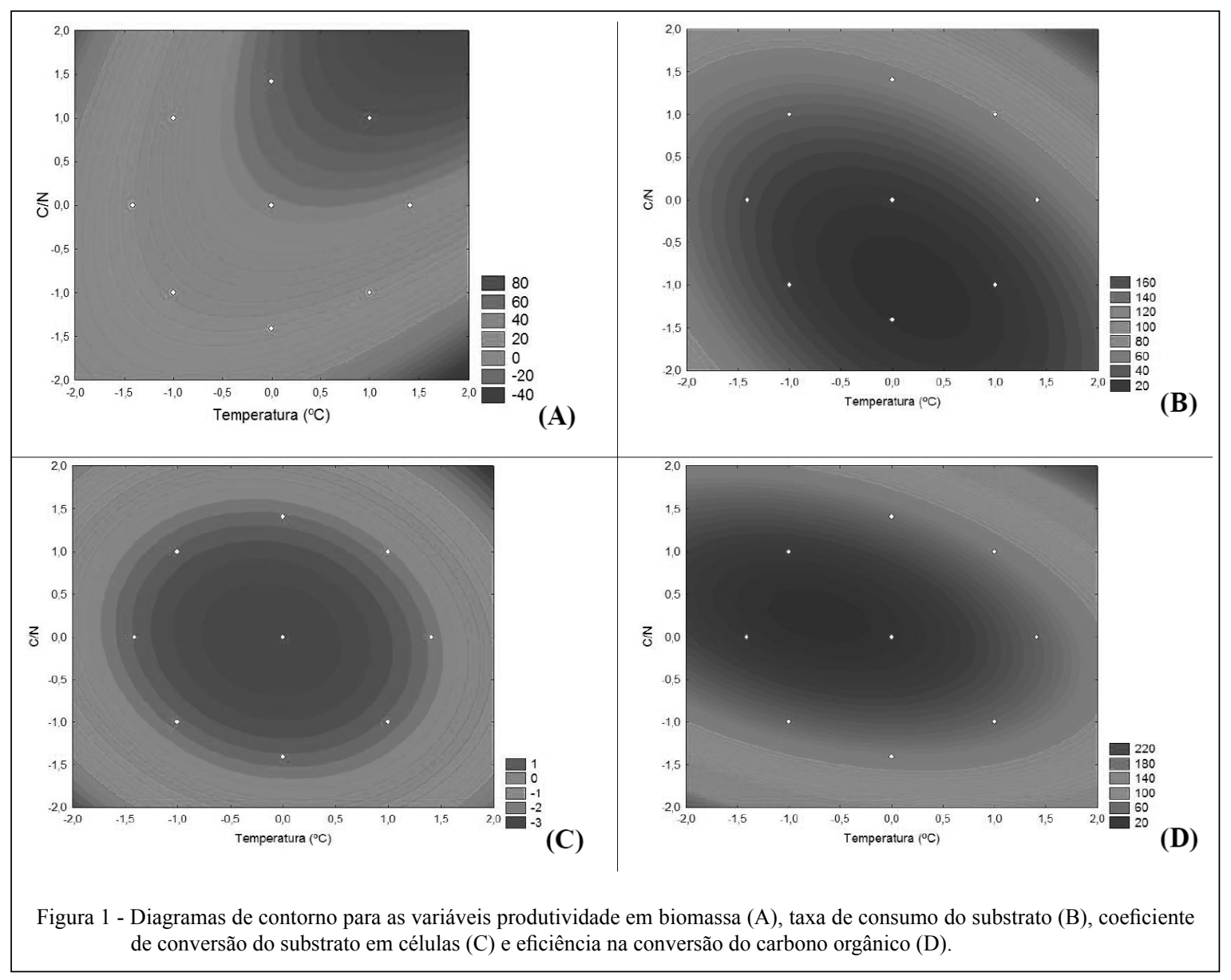

Ciência Rural, v.45, n.2, fev, 2015. 
Tabela 2 -Parâmetros cinéticos resultantes dos cultivos em biorreator e propriedades do biodiesel.

\begin{tabular}{|c|c|c|c|c|c|c|c|}
\hline Modo de Operação & $\mathrm{r}_{\mathrm{S}}\left(\mathrm{mg} \mathrm{L}^{-1} \mathrm{~h}^{-1}\right)$ & $\mathrm{Y}_{\mathrm{X} / \mathrm{S}}\left(\mathrm{mg}_{\text {célula }} \mathrm{mg}_{\text {amido }}{ }^{-1}\right)$ & $\mathrm{X}_{\text {máx }}\left(\mathrm{mgL}^{-1}\right)$ & $\mathrm{EC}(\%)$ & $\mathrm{P}_{\mathrm{X}}\left(\mathrm{mg} \mathrm{L}^{-1} \mathrm{~h}^{-1}\right)$ & Lipídeo(\%) & $P_{L}\left(m g ~ L^{-1} h^{-1}\right)$ \\
\hline Batelada & 91,43 & 0,55 & 4960 & 75,19 & 50,41 & 14,86 & 7,49 \\
\hline Batelada alimentada & 46,29 & 0,82 & 9200 & 41,66 & 42,13 & 10,17 & 4,28 \\
\hline \multirow{2}{*}{ Parâmetros biodiesel } & $\mathrm{EC}(\%)$ & $\mathrm{CN}$ & IS & II $\left(\mathrm{gI}_{2} 100 \mathrm{~g}^{-1}\right)$ & GI (\%) & FCC (\%) & $\operatorname{PEFF}\left({ }^{\circ} \mathrm{C}\right)$ \\
\hline & 99,99 & 54,88 & 406,82 & 21,47 & 23,88 & 12,48 & 39,21 \\
\hline
\end{tabular}

$\mathrm{r}_{\mathrm{S}}$ : taxa de consumo do substrato, $\mathrm{Y}_{\mathrm{X} / \mathrm{S}}$ : coeficiente de conversão do substrato em célula, $\mathrm{X}_{\text {máx }}$ : concentração celular máxima, EC: eficiência de conversão do carbono orgânico, $\mathrm{P}_{\mathrm{x}}$ : produtividade em biomassa, $\mathrm{P}_{\mathrm{L}}$ : produtividade lipídica, EC:conteúdo de éster, $\mathrm{CN}$ : número de cetano, IS: índice de saponificação, II: índice de iodo, GI: grau de instauração, FCC: fator de comprimento da cadeia, PEFF: ponto de entupimento de filtro a frio.

em batelada alimentada, por outro lado, apresentaram menor desempenho cinético para todos os demais parâmetros, com exceção do coeficiente de conversão do substrato em células $\left(0,82 \mathrm{mg}_{\text {células }} \mathrm{mg}_{\text {amido }}{ }^{-1}\right)$ e da concentração celular máxima $\left(9200 \mathrm{mgL}^{-1}\right)$, que foram substancialmente superiores quando comparadas aos cultivos em batelada. A definição da melhor estratégia de condução do bioprocesso, entretanto, foi realizada com base na produtividade em lipídeos, no qual o processo descontínuo demonstrou uma capacidade produtiva $75 \%$ superior ao processo descontínuo alimentado, como reflexo principalmente da baixa capacidade de acúmulo de óleo na célula nos cultivos descontínuos alimentados. De acordo com QUEIROZ et al. (2013), os lipídeos microbianos são produtos intracelulares e, desta forma, a produtividade lipídica global é obtida através da quantidade de lipídios da célula, multiplicada pela produtividade em biomassa, o que faz da produtividade em biomassa um critério primário na obtenção de lipídeos microbianos.

Estes resultados são potencialmente atrativos para a produção de óleos unicelulares por Phormidium sp., uma vez que este micro-organismo é capaz de converter eficientemente uma fonte orgânica de carbono abundantemente disponível no mercado internacional. Os processos similares, normalmente, são conduzidos a partir da hidrólise enzimática deste substrato, acarretando em operações unitárias adicionais, que impactarão nos custos operacionais do processo produtivo (LU et al., 2011; WEI et al., 2009).

Características do biodiesel

O perfil de ácidos graxos do óleo microalgal é o principal fator que determina as características do biodiesel (KNOTHE, 2005). Ao total, identificaramse quatro diferentes ácidos graxos majoritários: ácido caproico $(65,9 \%)$, ácido oleico $(23,88 \%)$, ácido caprílico $(5,43 \%)$ e ácido esteárico $(5,39 \%)$. Dessa forma, o perfil de ácidos graxos foi predominantemente saturado (76,11\%) e monoinsaturado (23,88\%). KNOTHE (2005) reporta que óleos com composição predominantemente saturada e monoinsaturada são os mais adequados para a síntese de biodiesel, pois produzem combustíveis com propriedades ideais ao uso em motores a diesel.

Com base nessa matriz oleaginosa, a síntese do biodiesel produziu um combustível (Tabela 2) com conteúdo de ésteres de 99,9\%, número de cetano de 54,88, índice de saponificação de 406,82, índice de iodo de $21,47 \mathrm{gI}_{2} 100 \mathrm{~g}^{-1}$, grau de instauração de $23,88 \%$, fator de comprimento da cadeia de $12,48 \%$ e ponto de entupimento de filtro a frio de $39,21^{\circ} \mathrm{C}$. As propriedades de combustão avaliadas são comparáveis ao biodiesel de soja (FRANCISCO et al., 2010) e estão de acordo com as normas Norte Americanas (ASTM 6751, 2002), da União Europeia (EN 14214, 2003) e brasileiras (ANP 255, 2003) para biodiesel, o que sugere a potencialidade de exploração do biodiesel obtido a partir da biomassa da Phormidium sp., nas condições avaliadas.

\section{CONCLUSÃO}

A produtividade em biomassa da microalga Phormidium sp. a partir de amido de mandioca é otimizada em razões $\mathrm{C} / \mathrm{N}$ de 68 e temperaturas de $30^{\circ} \mathrm{C}$. O cultivo em biorreator operado descontinuamente apresentou melhor desempenho na produção de óleos unicelulares $\left(7,49 \mathrm{mgL}^{-1} \mathrm{~h}^{-1}\right)$. O perfil de ácidos graxos foi predominantemente saturado $(76,11 \%)$, resultando em um biodiesel com propriedades de combustão (conteúdo de ésteres de 99,9\%, número de cetano de 54,88 , índice de iodo de $21,47 \mathrm{gI}_{2} 100 \mathrm{~g}^{-1}$, grau de instauração de $23,88 \%$ e ponto de entupimento de filtro a frio de $39,21^{\circ} \mathrm{C}$ ) dentro das principais normativas nacionais e internacionais. Nesse sentido, os resultados obtidos demonstram a potencialidade de exploração deste tipo de processo para a produção de biodiesel. 


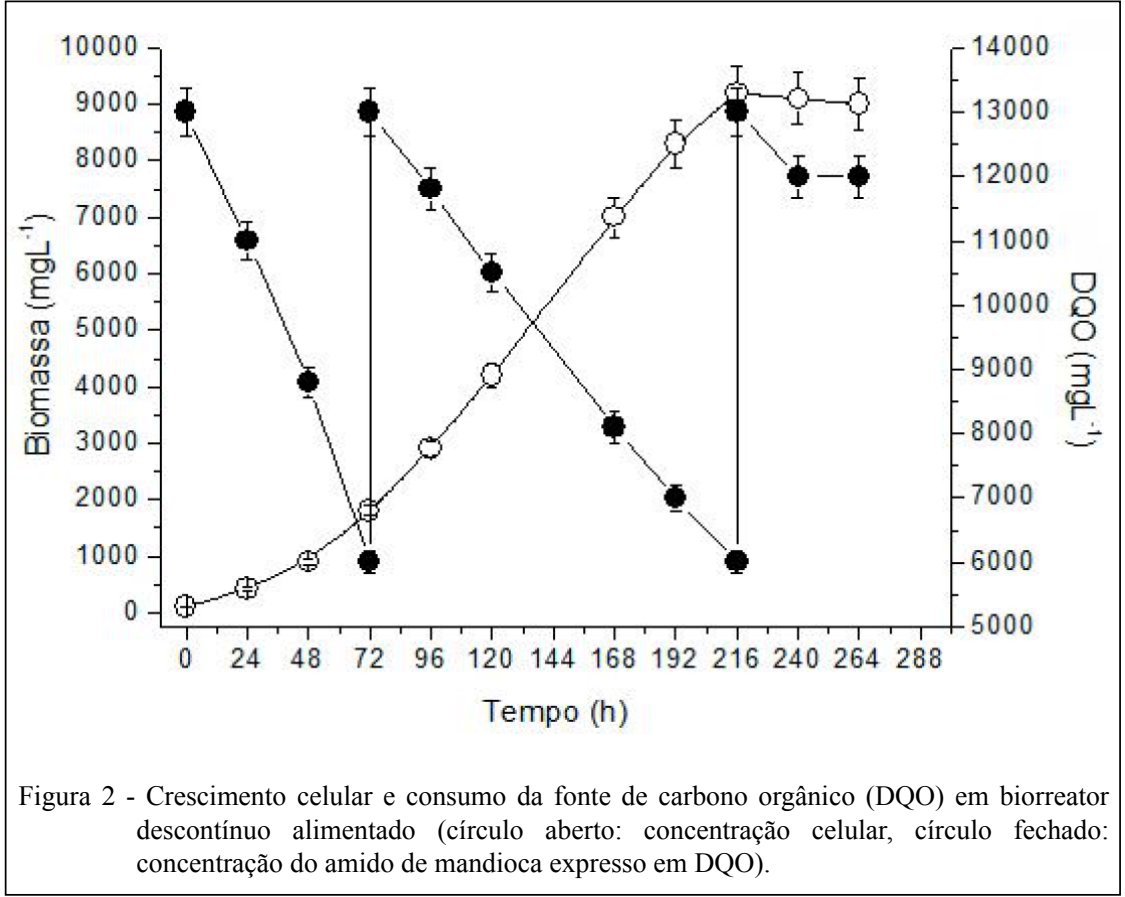

\section{AGRADECIMENTOS}

O financiamento para esta pesquisa foi fornecido pela Fundação de Amparo à Pesquisa do Estado de São Paulo (FAPESP).

\section{REFERÊNCIAS}

ANP (AGÊNCIA NACIONAL DO PETRÓLEO) 255. Provisional Brazilian Biodiesel Standard ANP. Brasil, 2003. Total de 21p.

APHA, AWWA, WEF (AMERICAN PUBLIC HEALTH ASSOCIATION, AMERICAN WATERWORKS ASSOCIATION, WATER ENVIRONMENTAL FEDERATION). Standard Methods for the Examination of Water and Wastewater. 21ed. Baltimore, Maryland: Prot City, 2005. 1325p.

ASTM 6751. Standard Specification for Biodiesel Fuel (B100). Blend Stock for Distillate Fuels. United States of America, 2002. In: KNOTHE, G. et al. (Ed.). The biodiesel handbook. Champaign, Illinois: AOCS, 2005. 332p.

BLIGH, E.G.; DYER, J.W. A rapid method of total lipid extraction and purification. Canadian Journal of Biochemistry and Physiology, v.37, n.8, p.911-917, 1959.Disponível em: $<$ http://www.nrcresearchpress.com/doi/abs/10.1139/o59099\#.U_OV2fldXaM>. Acesso em: 28 jan. 2010. doi: 10.1139/ 059-099.

FRANCISCO, E.C. et al. Microalgae as feedstock for biodiesel production: carbon dioxide sequestration, lipid production and biofuel quality. Journal of Chemistry Technology and Biotechnology, v.85, p.395-403, 2010. Disponível em: <http:// onlinelibrary.wiley.com/doi/10.1002/jctb.2338/abstract>. Acesso em: 29 jan. 2010 . doi: 10.1002/jctb.2338.
HADDAD, M.; FAWAZ, Z. Evaluation of microalgae alternative jet fuel using the AHP. Method with a Emphasis on the Environmental Progress \& Sustainable Energy, v.32, n.3, p.3044-3064, 2013. Disponível em: <http://onlinelibrary.wiley. com/doi/10.1002/ep.11638/abstract>. Acesso em: 06 fev. 2013. doi:10.1002/ep.11638.

HARTMAN, L.; LAGO, R.C.A. A rapid preparation of fatty acid methyl esters from lipids. Laboratory Pratice, v.22, p.475-476, 1976.

KRISNANGKURA, K. A Simple method for estimation of cetane index of vegetable oil methyl esters. JAOCS, v.63, n.4, p.552-553 1986. Disponível em: <http://link.springer.com/ article/10.1007\%2FBF02645752>. Acesso em: 29 jan. 2010.

LI, X. et al. Large-scale biodiesel production from microalga Chlorella protothecoides through heterotrophic cultivation in bioreactors. Biotechnology and Bioengineering, v.98, n.4, p.764-771, 2007. Disponível em: <http://www.ncbi.nlm.nih. gov/pubmed/17497732>. Acesso em: 29 jan. 2010. doi:10.1002/ bit.21489.

LU, Y et al. Simultaneous saccharification of cassava starch and fermentation of algae for biodiesel production. Journal Applied Phycology, 23, p.115-121, 2011. Disponível em: <http://link. springer.com/article/10.1007\%2Fs10811-010-9549-z>. Acesso em: 06 fev. 2013. doi: 07/s10811-010-9549-z.

MARTÍN, M.; GROSSMANN, I.E. On the synthesis of sustainable biorefineries. Industrial \& Engineering Chemistry Research, v.52, p.3044-3064, 2012. Disponível em: <http://pubs.acs.org/doi/ abs/10.1021/ie2030213 >. Acesso em: 06 fev. 2013. doi: 10.1021/ ie2030213.

MATA, T.M. et al. Microalgae for biodiesel production and their applications: a review. Renewable \& Sustainable Energy 
Reviews, v.14, p.217-232, 2010. Disponível em: <http://www. sciencedirect.com/science/article/pii/S1364032109001646>. Acesso em: 29 jan. 2010. doi: 10,1016/j.rser.2009.07.020.

MOHAMED, M.S. et al. Heterotrophic cultivation of microalgae for production of biodiesel. Recent Patents on Biotechnology, v.5, p.95-107, 2011. Disponível em: <http:/www.ncbi.nlm.nih.gov/ pubmed/21707527>. Acesso em: 06 fev. 2013. doi:10.2174/10095.

PEREZ-GARCIA, O. et al. Heterotrophic cultures of microalgae: metabolism and potential products. Water Research, v.45, p.1136, 2011. Disponível em: <http://www.sciencedirect.com/science/ article/pii/S0043135410006019>. Acesso em: 06 fev. 2013. doi:10.1016/j.watres.2010.08.037.

QUEIROZ, M.I. et al. Fish processing wastewater as a platform of the microalgal biorefineries. Biosystems Engineering, v.115, p.195-202, 2013. Disponível em: <http:/www.sciencedirect.com/ science/article/pii/S1537511012002243>. Acesso em: $06 \mathrm{fev}$. 2013. doi:10.1016/j.biosystemseng.2012.12. 013.

RAMOS, M.J. et al. Influence of fatty acid composition of raw materials in biodiesel properties. Bioresource Technology, v.100, p.261-268, 2009. Disponível em: <http://www.sciencedirect.com/ science/article/pii/S0960852408005464>. Acesso em: 29 jan. 2010. doi: 10.1016/j.biotech.2008.06.039.

RIPPKA, R. et al. Generic assignments strain histories and properties of pure cultures of cyanobacteria. Journal of General Microbiology, v.111, n.1, p.61, 1979. Disponível em: <http://mic. sgmjournals.org/content/111/1/1.short>. Acesso em: 29 jan. 2010 doi:10.1099/00221287-111-1-1.
STATSOFT, Inc.STATISTICA for Windows [Computer program manual]. [Online]. Statsoft Inc., Tulsa, Oklahoma, 2004. Disponível em: <http://www.statsoftinc.com>. Acesso em: 29 jan. 2010 .

SUALI, E.; SARBATLY, R. Conversion of microalgae to biofuel. Renewable and Sustainable Energy Reviews, v.16, p.4316-4342, 2012. Disponível em: <http://www.sciencedirect.com/science/ article/pii/S1364032112002304>. Acesso em: 06 fev. 2013. doi: 10.1016/j.rser.2012.03.047.

UNE-EN 14214. Automotive Fuels, Fatty Acid Methyl Esters (FAME) for Diesel Engines, Requirements and TestMethods. European Union, 2003. 15p. In: KNOTHE, G. et al. (Ed.). The biodiesel handbook. Champaign, Illinois: AOCS, 2005. 332p

$\mathrm{XU}, \mathrm{H}$. et al. High quality biodiesel production from a microalga Chlorella protothecoides by heterotrophic growth in fermenters. Journal of Biotechnology, v.126, p.499-507, 2006. Disponível em: <http://www.ncbi.nlm.nih.gov/pubmed/16772097>. Acesso em: 06 fev. 2013. doi: 1016/j.jbiotec.2006.05.002.

WEI, A. et al. Effects of cassava starch hydrolysate on cell growth and lipid accumulation of the heterotrophic microalgae Chlorella protothecoides. Journal of Industrial Microbiology and Biotechnology, v.36, p.1383-1389, 2009. Disponível em: <http:// www.ncbi.nlm.nih.gov/pubmed/19633877>. Acesso em: 29 jan. 2010. doi: 10.1007/s10295-009-0624-x.

KNOTHE, G. Fuel properties. In: KNOTHE, G. et al. (Ed.). The biodiesel handbook. Champaign, Illinois: AOCS, 2005. 332p. 